\title{
Effect of contaminations and surface preparation on the work function of single layer $\mathrm{MoS}_{\mathbf{2}}$
}

\author{
Oliver Ochedowski ${ }^{1}$, Kolyo Marinov ${ }^{1}$, Nils Scheuschner ${ }^{2}$, Artur Poloczek ${ }^{3}$, \\ Benedict Kleine Bussmann ${ }^{1}$, Janina Maultzsch ${ }^{2}$ and Marika Schleberger ${ }^{* 1}$
}

Open Access

\author{
Full Research Paper \\ Address: \\ ${ }^{1}$ Fakultät für Physik and CeNIDE, Universität Duisburg-Essen, \\ Lotharstr. 1, 47057 Duisburg, Germany, ${ }^{2}$ Institut für Festkörperphysik, \\ Technische Universität Berlin, Hardenbergstr. 36, 10623 Berlin, \\ Germany and ${ }^{3}$ Solid State Electronics Department and CeNIDE, \\ University of Duisburg-Essen, Lotharstr. 55, 47058 Duisburg, \\ Germany \\ Email: \\ Marika Schleberger* - marika.schleberger@uni-due.de \\ ${ }^{*}$ Corresponding author \\ Keywords: \\ KPFM; $\mathrm{MoS}_{2}$; NC-AFM; surface potential; work function
}

Beilstein J. Nanotechnol. 2014, 5, 291-297.

doi:10.3762/bjnano.5.32

Received: 31 October 2013

Accepted: 11 February 2014

Published: 13 March 2014

This article is part of the Thematic Series "Noncontact atomic force microscopy II".

Guest Editors: U. D. Schwarz and M. Z. Baykara

(C) 2014 Ochedowski et al; licensee Beilstein-Institut.

License and terms: see end of document.

\begin{abstract}
Thinning out $\mathrm{MoS}_{2}$ crystals to atomically thin layers results in the transition from an indirect to a direct bandgap material. This makes single layer $\mathrm{MoS}_{2}$ an exciting new material for electronic devices. In $\mathrm{MoS}_{2}$ devices it has been observed that the choice of materials, in particular for contact and gate, is crucial for their performance. This makes it very important to study the interaction between ultrathin $\mathrm{MoS}_{2}$ layers and materials employed in electronic devices in order to optimize their performance. In this work we used NC-AFM in combination with quantitative KPFM to study the influence of the substrate material and the processing on single layer $\mathrm{MoS}_{2}$ during device fabrication. We find a strong influence of contaminations caused by the processing on the surface potential of $\mathrm{MoS}_{2}$. It is shown that the charge transfer from the substrate is able to change the work function of $\mathrm{MoS}_{2}$ by about $40 \mathrm{meV}$. Our findings suggest two things. First, the necessity to properly clean devices after processing as contaminations have a great impact on the surface potential. Second, that by choosing appropriate materials the work function can be modified to reduce contact resistance.
\end{abstract}

\section{Introduction}

Due to their unique properties which can differ a lot compared to bulk materials, two-dimensional materials are being targeted in a variety of research areas like surface physics, electrical engineering, chemistry and biomedical applications [1-4]. The 2D-material getting the most attention besides graphene are single layers of molybdenum disulfide (SLM) which consist of a plane of molybdenum atoms that are sandwiched between sulfur atoms. The main reason for this is the transition from an indirect (bulk $\mathrm{MoS}_{2}$ ) to a direct (single layer $\mathrm{MoS}_{2}$ ) band gap semi-conductor [5]. Single layer $\mathrm{MoS}_{2}$ has a strong photoluminescence signal [5-9] and other interesting properties like a mechanical stiffness of $180 \pm 60 \mathrm{~N} \cdot \mathrm{m}^{-1}$, which is comparable to steel $[10,11]$, charge carrier mobilities that are comparable to $\mathrm{Si}$ $[12,13]$, and it is possible to grow these ultrathin layers using 
CVD [14-16]. The main advantage SLM has to offer compared to the model 2D-material graphene is its direct band gap. It allows the facile integration of SLM in electronic devices, which has been demonstrated for highly flexible transistors, optoelectronic devices, small-signal amplifiers, $\mathrm{MoS}_{2}$ integrated circuits and chemical vapor sensors [12,17-21]. It has been reported that the performance of these devices can greatly vary due to the choice of the material of the contacts, the cleanliness of the SLM surface and a top gated structure with a high $\kappa$ dielectric [22-27]. By choosing appropriate materials in 2D-devices the work function can be tuned to, e.g., lower the contact resistance and improve their performance. First experiments adressing this issue for $\mathrm{MoS}_{2}$ by using Kelvin probe force microscopy (KPFM) have already been reported [28,29]. However, these measurements were not done on SLM but bilayer $\mathrm{MoS}_{2}$ (BLM) and higher layer numbers and the measurements were performed under ambient conditions using amplitude modulated KPFM, both having a great impact on the results. In this work we study the work function of SLM on a standard $\mathrm{SiO}_{2} / \mathrm{Si}$ substrate using non-contact atomic force microscopy (NC-AFM) and Kelvin probe force microscopy in situ. In our measurements we use a gold contact patterned on SLM in order to calibrate the work function of our AFM tip which allows us to determine quantitative work function values for SLM, BLM and few layer $\mathrm{MoS}_{2}$ (FLM). Additionaly, we use reactive ion etching to pattern holes into the $\mathrm{SiO}_{2}$ substrate. By comparing the work function of SLM on etched and pristine $\mathrm{SiO}_{2}$ substrates, we show that a significant change in the work function can be achieved by substrate effects.

\section{Experimental}

For our studies we exfoliated $\mathrm{MoS}_{2}$ (HQgraphene, Netherlands) on a patterned $\mathrm{Si}$ sample that has been covered by $90 \mathrm{~nm} \mathrm{\textrm {SiO } _ { 2 }}$ layer (graphene supermarket, Calverton, NY, USA). The $\mathrm{SiO}_{2}$ was patterned by using an inductive coupled plasma reactive ion etching (ICP-RIE) with $\mathrm{Cl}_{2} / \mathrm{N}_{2}$ chemistry. The etching mask used was a standard photoresist patterned by optical lithography. The etching was performed at $35^{\circ} \mathrm{C}$ using $300 \mathrm{~W}$ of ICP and $150 \mathrm{~W}$ table power. The chamber pressure was adjusted to $8 \cdot 10^{-3}$ mbar during this procedure. Reactive ion etching was employed to locally alter the surface roughness and introduce defects in the $\mathrm{SiO}_{2}$ substrate [30,31]. The resulting structures on the $\mathrm{SiO}_{2}$ surface consist of etched holes with a depth of about $40 \mathrm{~nm}$ measured using AFM. Immediately after etching, the $\mathrm{MoS}_{2}$ was exfoliated by mechanical cleavage [32]. Single layer $\mathrm{MoS}_{2}$ flakes were located by using their optical contrast and verified using Raman spectroscopy [33,34]. For Raman point measurements and mappings, a Renishaw InVia Raman spectrometer $(\lambda=532 \mathrm{~nm}, P<0.4 \mathrm{~mW}$, spectral resolution $\approx 1 \mathrm{~cm}^{-1}$ ) has been employed. Because SLM is highly flexibel, it is not covering the etched hole. Instead the
SLM touches the etched $\mathrm{SiO}_{2}$ surface at the bottom and follows the morphology like a membrane (Figure 1). While this leaves the SLM heavily strained on the edge of the hole, it allows to experimentally compare the effect of two differently treated subtrates $\left(\mathrm{SiO}_{2}\right.$ and RIE $\left.\mathrm{SiO}_{2}\right)$ on the same $\mathrm{MoS}_{2}$ flake. After identification of SLM areas, a Ti/Au $(5 \mathrm{~nm} / 15 \mathrm{~nm})$ contact was patterned on the $\mathrm{MoS}_{2}$ flake by photolithography. We used the Photoresist ARP-5350 (Allresist GmbH, Strausberg, Germany) with the developer AR 300-35 (Allresist GmbH, Strausberg, Germany). Acetone was used for the lift-off and finally the samples were boiled in isopropyl alcohole. The contact served two purposes. On the one hand, the sample was electrically connected to ground potential, on the other hand, the gold surface was used for calibrating the work function of the AFM tip during KPFM measurements.

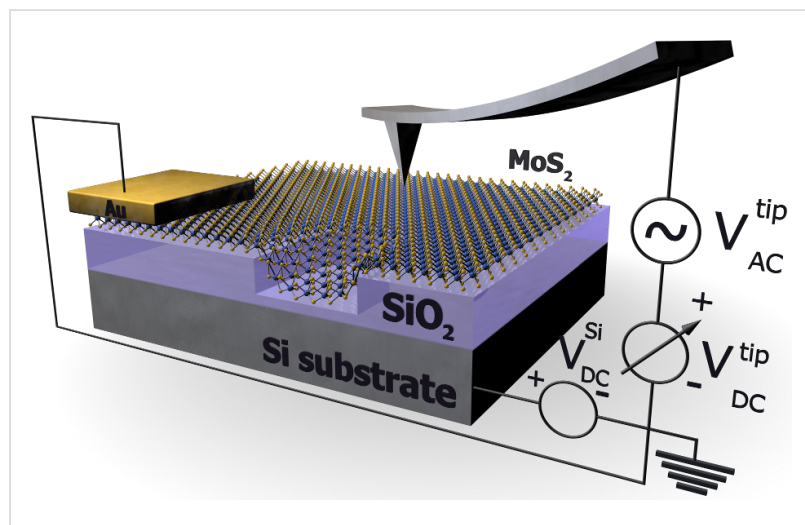

Figure 1: Schematic representation of the KPFM setup and the $\mathrm{MoS}_{2}$ sample with the RIE $\mathrm{SiO}_{2}$.

The contacted SLM sample is introduced into an ultra high vacuum system with a base pressure of about $2 \cdot 10^{-10} \mathrm{mbar}$. Non-contact AFM measurements were performed using a RHK UHV 7500 system with the PLL Pro 2 controller. Simultaneously to NC-AFM, frequency-modulated KPFM measurements were conducted to probe the local contact potential difference (CPD) between the tip and the surface [35-41]. As force sensors, highly conductive Si cantilevers with a typical resonance frequency of $f=300 \mathrm{kHz}$ (Vistaprobe T300) were utilized. During KPFM measurements an AC voltage is applied to the tip $\left(U_{\mathrm{AC}}=1 \mathrm{~V}\right.$ and $\left.f_{\mathrm{AC}}=1 \mathrm{kHz}\right)$ and the built in lock-in amplifier of the PLL Pro 2 is used to apply a DC voltage which minimizes the resulting electrostatic forces between tip and sample surface. This DC voltage corresponds to the local CPD.

\section{Results and Discussion \\ Raman spectroscopy characterization}

In Figure 2 we present an optical image of a sample prepared by the procedure described above together with additional Raman spectroscopy data. The SLM flake can be identified in the 

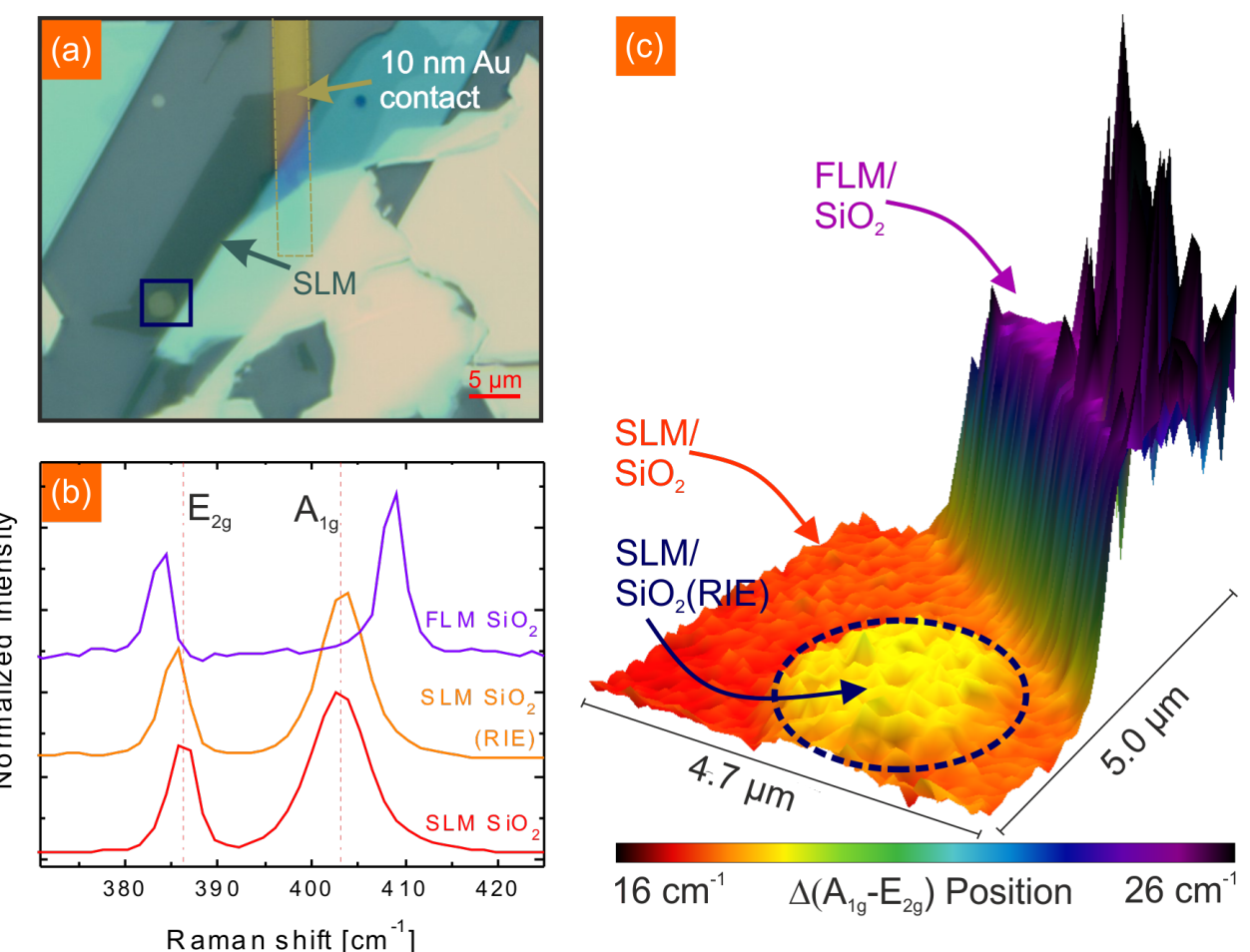

Figure 2: (a) Optical microscope image of an exfoliated $\mathrm{MoS}_{2}$ flake on a prepatterned (RIE) $\mathrm{SiO}_{2}$ substrate. A gold contact was attached to the MoS 2 in order to ground the flake for KPFM measurements. (b) Raman spectroscopy spectra of SL and FL MoS on $_{2} \mathrm{SiO}_{2}$ and $\mathrm{SL} \mathrm{MoS}_{2}$ on RIE SiO . For higher layer numbers the $E_{2 g}$ is shifted to lower wave number while the $A_{1 g}$ mode is shifted to higher wave numbers. (c) Raman mapping data of the area marked in (a) with the blue box. The difference between $A_{1 g}$ and $E_{2 g}$ mode is plotted revealing a shift of the Raman modes for SLM on the RIE $\mathrm{SiO}_{2}$ substrate.

optical image in Figure 2a by its contrast, which is a transparent green tone. While the majority of the SLM flake is located on pristine $\mathrm{SiO}_{2}$, a small part of the SLM flake is at the bottom of a hole which was patterned by RIE. To unambiguously identify SLM we used Raman spectroscopy and compared the results to data obtained by literature [34]. In Figure $2 b$ the Raman spectra of SLM on $\mathrm{SiO}_{2}$ and on $\mathrm{SiO}_{2}$ (RIE) as well as FLM on $\mathrm{SiO}_{2}$ is shown. The two prominent peaks, the $E_{2 g}$ and $A_{1 g}$ peak, correspond to the opposite vibration of the two $\mathrm{S}$ atoms with respect to the Mo atom and the out-of-plane vibration of only $\mathrm{S}$ atoms in opposite directions, respectively [42,43]. For SLM on $\mathrm{SiO}_{2}$ the Raman shifts obtained for the $E_{2 g}$, $v=386.1 \mathrm{~cm}^{-1}$, and $A_{1 g}, v=403.0 \mathrm{~cm}^{-1}$, are consistent with values reported by other groups. For higher layer numbers the $E_{2 g}$ has been reported to shift to lower wave numbers while the $A_{1 g}$ shifts to larger wave numbers which is again in good agreement with our data. However, the SLM on RIE $\mathrm{SiO}_{2}$ shows a different behaviour compared to SLM on pristine $\mathrm{SiO}_{2}$. The $E_{2 g}$ is slightly downshifted to $v=385.2 \mathrm{~cm}^{-1}$ and the $A_{1 \mathrm{~g}}$ shows a minor shift to $v=403.4 \mathrm{~cm}^{-1}$. Shifts of the $E_{2 g}$ and $A_{1 g}$ modes of SLM can have multiple reasons. Uniaxial tensial strain has been observed to cause a splitting in the $E_{2 g}$ mode and a shift to lower wave numbers for the resulting $E^{-}$and $E^{+}$modes by 4.5 and $1 \mathrm{~cm}^{-1} / \%[44,45]$. While the $A_{1 g}$ mode shows no distinct sensitivity to uniaxial strain, a charge carrier dependency has been observed [46]. Electron doping of $1.8 \cdot 10^{13} \mathrm{~cm}^{-2}$ leads to a linewidth broadening of $6 \mathrm{~cm}^{-1}$ and the phonon frequency decreases by $4 \mathrm{~cm}^{-1}$. As our data shows a shift in both Raman active modes we suggest that the RIE $\mathrm{SiO}_{2}$ surface causes a slight strain and maybe local doping by charge transfer in the $\mathrm{MoS}_{2}$ flake. The Raman mapping shown in Figure 2c corresponds to the evaluation of point spectra performed in the green box marked in Figure 2a. Plotted is the difference of the $E_{2 g}$ an $A_{1 g}$ mode positions. While the difference between SLM and FLM on $\mathrm{SiO}_{2}$ is significant with $\Delta=8.2 \mathrm{~cm}^{-1}$, the difference

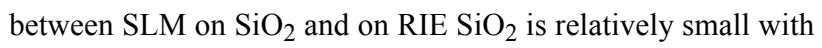
$\Delta=1.3 \mathrm{~cm}^{-1}$. As can be seen in the Raman mapping, the difference in the SLM induced by the substrate is constant over the whole flake and not just present in single point meaurements.

\section{In-situ KPFM on single layers of $\mathrm{MoS}_{2}$}

For the NC-AFM and KPFM measurements the sample was introduced to the UHV system. Before the data collection the sample was heated in situ to $200{ }^{\circ} \mathrm{C}$ for $30 \mathrm{~min}$ to remove any 
adsorbates from ambience. In Figure $3 \mathrm{a}$ and Figure $3 \mathrm{c}$ the NC-AFM topography and the corresponding surface potential map are shown, respectively. On the right side the $\mathrm{Ti} / \mathrm{Au}$ contact can be seen which is about $20 \mathrm{~nm}$ high and shows a distinct contrast in the surface potential in comparison to the $\mathrm{MoS}_{2}$ layers. In Figure 3d a surface potential histogram of SLM, FLM and the gold surface of the Ti/Au contact is given. We find a surface potential of $4.27 \mathrm{~V}$ for SLM, 4.37 V for FLM and $4.89 \mathrm{~V}$ for gold. The surface potential itself is always a relative value based on the local CPD between the AFM tip and the sample surface. To obtain quantitative work function values, we calibrated the tip on the gold surface by using the known work function of gold $\Phi_{\mathrm{Au}}=5.10 \mathrm{eV}[47,48]$. With the relation $\Phi=5.10 \mathrm{eV}-e \cdot\left(\mathrm{CPD}_{\mathrm{Au}}-\mathrm{CPD}_{\mathrm{nMoS} 2}\right)$ the work function of $\mathrm{SLM} \Phi_{\mathrm{SLM}}=4.49 \pm 0.03 \mathrm{eV}$ and FLM $\Phi_{\mathrm{FLM}}=4.59 \pm 0.03 \mathrm{eV}$ can be assigned. The given errorbar consists of the experimental error of our system. Not included in this error is band bending, which occurs when doing KPFM measurements on a semi-conductor surface and a false estimation of the work function of the patterned gold contact. Besides graphite [49], gold is a common material to calibrate the work function of the AFM tip [48], but while the work function $\Phi_{\mathrm{Au}}=5.10 \mathrm{eV}$ is often used, other work function values in the range from $4.74 \mathrm{eV}$ to

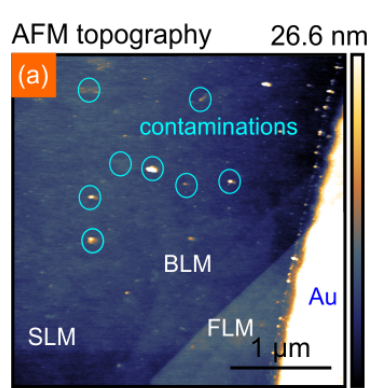

$0.00 \mathrm{~nm}$

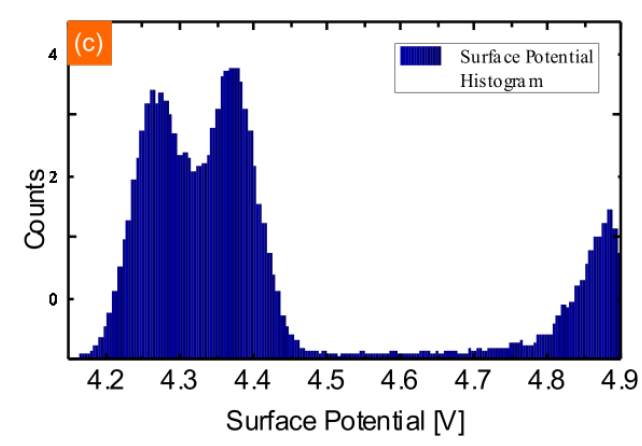

Figure 3: (a) NC-AFM image of $\mathrm{MoS}_{2}$ flake on $\mathrm{SiO}_{2}$ with a gold contact (height $=20 \mathrm{~nm}$ ). Topography shows areas with contaminations due to processing. (b) Corresponding surface potential image to (a). The surface potential of $\mathrm{MoS}_{2}$ is increasing with increasing layer thickness, contaminations can be clearly distinguished in the surface potential image. (c) Surface potential histogram of the box marked in (b).
$5.54 \mathrm{eV}$ have been reported as well [50,51]. Surface roughness, homogeneity and humidity can have an effect on the measured work function of metal surfaces as Guo et al. recently demonstrated [52]. The presented data is measured in situ after annealing and we are therefore confident that humidity can be neglected. We want to point out that an error in the work function calibration does not affect the work function values of SLM, BLM and FLM with respect to each other. While the surface potential on the Au contact in Figure 3 appears uniform, strong local variations can be observed on the $\mathrm{MoS}_{2}$ flake. We attribute these features, marked in Figure $3 \mathrm{a}$ with green circles, to contaminations due to the patterning process. The height of these contaminations varies between $1 \mathrm{~nm}$ and $20 \mathrm{~nm}$. These contaminations have a noticeable effect on the work function of SLM, as $\Phi_{\text {SLM }}$ can be lowered by up to $0.15 \mathrm{eV}$. As the work function of these contaminations is clearly different than that of the $\mathrm{Au}$ contact, the contaminations are most likely resist residues which have not been completely removed. Such contaminations may act as scattering centers or charge puddles which are likely to be detrimental to the performance of SLM devices [53]. For graphene and $\mathrm{MoS}_{2}$ it has been shown, that adsorbates due to ambient exposure can have a strong impact on the work function of these materials, like inducing an additional charge transfer or even redox reactions with water [29,54].

\section{In situ screening length of $\mathrm{MoS}_{2}$}

In the next step, we determine the work function of BLM and the screening length of $\mathrm{MoS}_{2}$. For this the SLM/BLM/FLM section of Figure 3 has been measured again in more detail and the work function is analyzed by line profiles. Shown in Figure $4 \mathrm{a}-\mathrm{c}$ are the NC-AFM topography, work function map and the corresponding line profiles, respectively. The measured height for BLM is $0.92 \pm 0.10 \mathrm{~nm}$, which is slightly higher than the interlayer spacing of a bulk $\mathrm{MoS}_{2}$ crystal [55]. For FLM we get two different heights, one is $2.96 \mathrm{~nm}(\approx 5$ layers $)$ and $7.89 \mathrm{~nm}(\approx 12-13$ layers $)$. In the work function map in Figure $4 \mathrm{~b}$, three contrasts can be observed - SLM, BLM and FLM. As the work function for FLM $2.96 \mathrm{~nm}$ and the other FLM with $7.89 \mathrm{~nm}$ is not changing, we conclude from our data that the screening length of $\mathrm{MoS}_{2}$ is at least $2.96 \mathrm{~nm}$, which is in good agreement with previous findings for annealed $\mathrm{MoS}_{2}$ [29]. Li et al. compared the screening length of pristine $\mathrm{MoS}_{2}$ flakes on $\mathrm{SiO}_{2}$ with annealed $\mathrm{MoS}_{2}$ flakes and found a decrease from approximately $5 \mathrm{~nm}$ down to $2.5 \mathrm{~nm}$ for annealed $\mathrm{MoS}_{2}$. Our measurements here yield a screening length between 1.6 and $2.96 \mathrm{~nm}$, which is much lower than the value for pristine $\mathrm{MoS}_{2}$. We therefore conclude that the investigated $\mathrm{MoS}_{2}$ is not affected by ambient adsorbates. In Figure $4 c$ we used the line profile to quantify the work function of SLM and BLM. The work function of SLM is determined to be the same as using the histogram analysis in Figure 3 with $\Phi_{\mathrm{SLM}}=4.49 \pm 0.03 \mathrm{eV}$. 


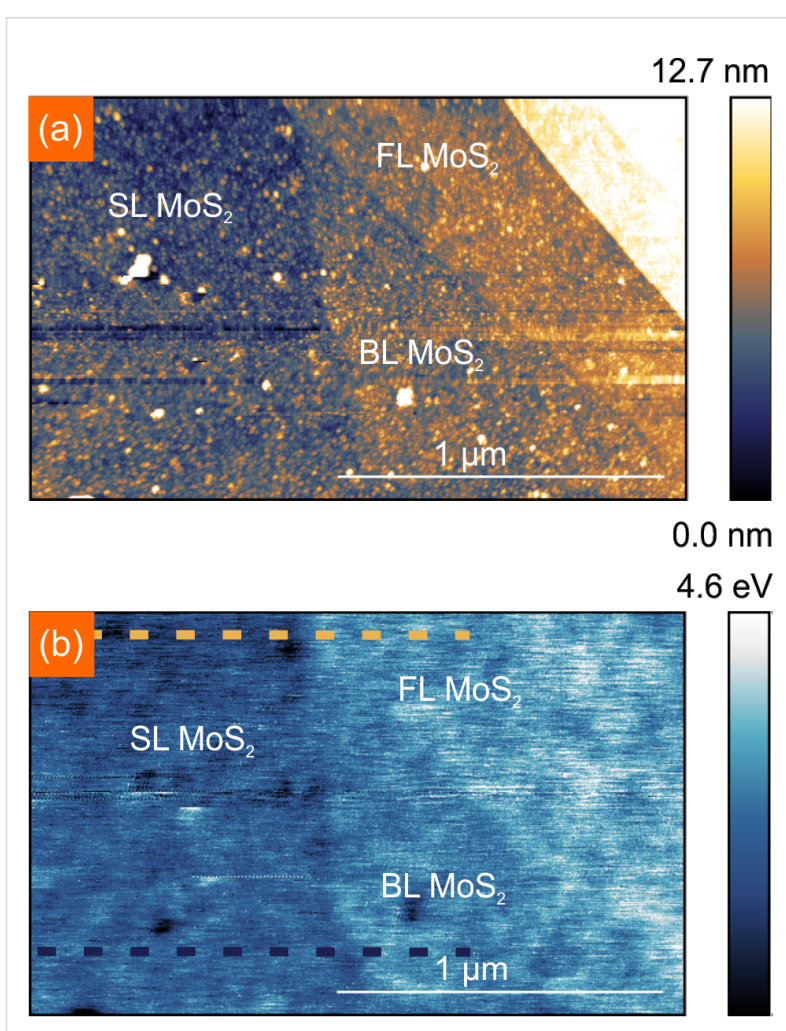

(c)

$4.4 \mathrm{eV}$

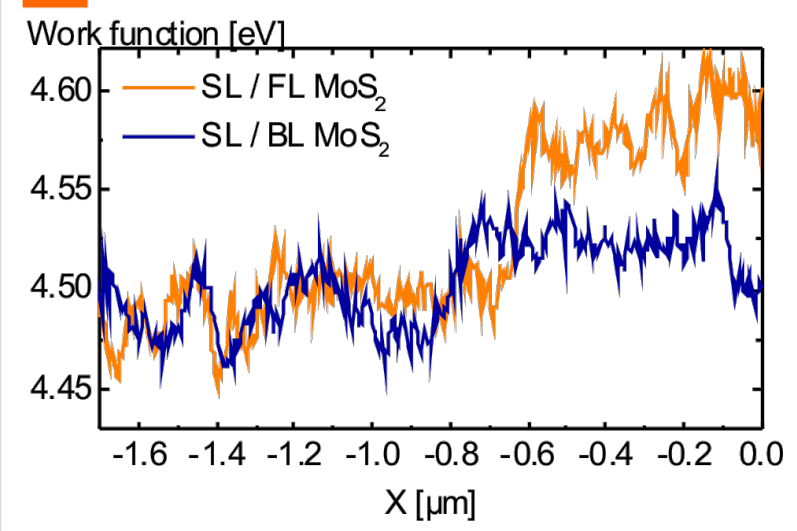

Figure 4: (a) NC-AFM zoom-in of an area consisting of $1 \mathrm{~L}, 2 \mathrm{~L}$ and $\mathrm{FL}$ $\mathrm{MoS}_{2}$. (b) Corresponding KPFM image, calibration of the tip on the gold contact allows assignment of work functions to surface potential values. Plotted is the work function. (c) Line profiles of the work function corresponding to the lines marked in (b).

The work function of BLM is increased with respect to SLM by about $0.05 \mathrm{eV}$ to $\Phi_{\mathrm{BLM}}=4.54 \pm 0.03 \mathrm{eV}$. Again, contaminations on BLM appear to decrease the work function as can be seen in Figure $4 b$.

\section{Substrate effects on the work function of single layer $\mathrm{MoS}_{2}$}

To study the effect of the substrate on the work function of SLM, we compare the work function of $\mathrm{SLM}$ on $\mathrm{SiO}_{2}$ with
SLM in the RIE $\mathrm{SiO}_{2}$ holes in Figure 5. The work function map in Figure $5 \mathrm{~b}$ shows an increased work function over the etched hole of about $\Delta \Phi=0.04 \mathrm{eV}$. This shift is caused by the charge transfer from the etched substrate which leads to an effective doping that has been proven to have a large impact on the optical properties of SLM [56]. The etched $\mathrm{SiO}_{2}$ substrate has an effect on the surface potential distribution as well. By comparing histogram data of $\mathrm{SLM}$ on $\mathrm{SiO}_{2}$ and $\mathrm{RIE} \mathrm{SiO}_{2}$ (see inset in Figure 5c) we find a decreased surface potential fluctuation by $0.02 \mathrm{eV}$ for SLM on the etched $\mathrm{SiO}_{2}$. The potential fluctuation is related to charge impurities which are detrimental for the performance of 2D-devices and KPFM is an efficient way to probe it [57]. Further, a lower potential fluctuation indicates a higher charge homogeneity. Charge inhomogeneity has been shown to play a crucial role in the oxidative reactivity of graphene [58]. At the edge of the etched hole, where SLM is heavily bent, a strong increase in the work function by another $\Delta \Phi=0.05 \mathrm{eV}$ compared to $\mathrm{SLM}$ on the $\mathrm{RIE} \mathrm{SiO}_{2}$ substrate caused by stress can be observed. It has been shown by Castellanos-Gomez et al. that heavy strain in SLM has a large impact on the band gap of SLM [59]. However, KPFM only measures the contact potential difference (from which we derive the work function). For insulating materials there is no straightforward relation between the contact potential difference and the band-gap. Therefore, our results are not directly comparable. The plot in Figure 5c sums up our findings with respect to the work function of $\mathrm{MoS}_{2}$. The work function of FLM in ambient has been determined previously by amplitude modulated KPFM. The reported values of $\Phi=5.25 \mathrm{eV}$ [28] are significantly higher than the values found here. This difference is clearly due to the contaminations which are absent in our measurements. Our data should instead be compared to the values determined by other means like ultraviolet photoelectron spectrosocopy [60-63]. The excellent agreement again underlines the importance of UHV measurements if intrinsic properties are to be probed.

\section{Conclusion}

In conclusion we have performed the first in situ Kelvin probe force microscopy measurements on single layers of $\mathrm{MoS}_{2}$ on a $\mathrm{SiO}_{2}$ substrate. We find work functions of $\Phi_{\mathrm{SLM}}=4.49 \mathrm{eV}$, $\Phi_{\mathrm{BLM}}=4.54 \mathrm{eV}$ and $\Phi_{\mathrm{FLM}}=4.59 \mathrm{eV}$ for SLM, BLM and FLM respectively. We observe a screening length between 1.6 and $3.5 \mathrm{~nm}$ which indicates a clean $\mathrm{MoS}_{2}$ flake. We have further investigated the effect of the substrate on the work function of $\mathrm{MoS}_{2}$ by partly etching the $\mathrm{SiO}_{2}$ substrate. Raman spectroscopy measurements suggests substrate effects like strain which increase the work function of SLM of $\Delta \Phi=0.04 \mathrm{eV}$ on etched $\mathrm{SiO}_{2}$. The next step is to investigate completely free standing $\mathrm{MoS}_{2}$ flakes without a substrate in order to probe the intrinsic charge homogeneity and work function of SLM. 

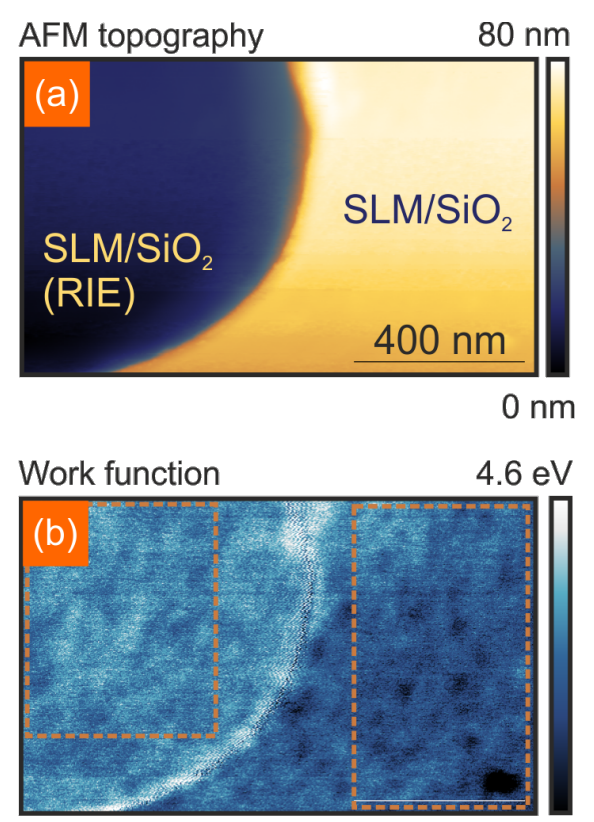

$4.4 \mathrm{eV}$

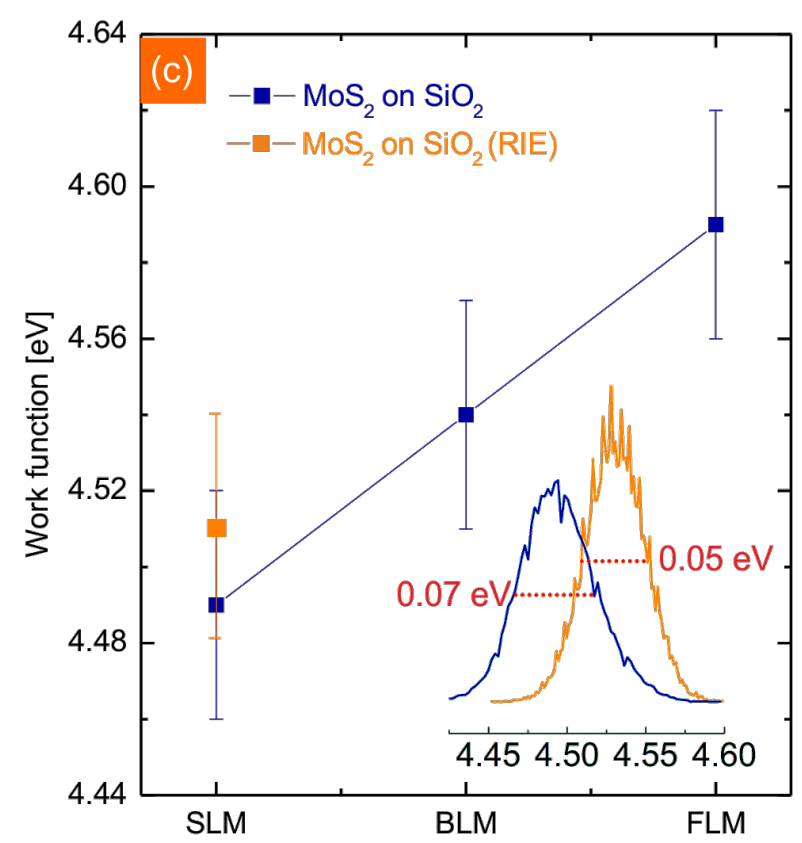

Figure 5: (a) NC-AFM topography of $\mathrm{SLM}$ on $\mathrm{SiO}_{2}$ and holes etched in $\mathrm{SiO}_{2}$ using RIE. (b) Work function map corresponding to the topography shown in (a). The work function of SLM on etched $\mathrm{SiO}_{2}$ is increased compared to pristine $\mathrm{SiO}_{2}$. (c) Layer dependent work function of $\mathrm{MoS}_{2}$. The inset shows the work function histogram evaluation of the areas marked in (b). The FWHM of SLM on RIE $\mathrm{SiO}_{2}$ is decreased by $0.02 \mathrm{eV}$.

\section{Acknowledgements}

We acknowledge financial support from the DFG in the framework of the Priority Program 1459 Graphene (O.O., N.S.), the SFB 616 Energy dissipation on surfaces (K.M., B.K.B.), and from the ERC under Grant No. 259286 (J.M.). We thank M. Freudenberg for graphics support.

\section{References}

1. Geim, A. K. Science 2009, 324, 1530-1534. doi:10.1126/science. 1158877

2. Novoselov, K. S.; Fal'ko, V. I.; Colombo, L.; Gellert, P. R.; Schwab, M. G.; Kim, K. Nature 2012, 490, 192. doi:10.1038/nature11458

3. Xu, M.; Liang, T.; Shi, M.; Chen, H. Chem. Rev. 2013, 113, 3766 doi:10.1021/cr300263a

4. Shen, H.; Zhang, L.; Liu, M.; Zhang, Z. Theranostics 2012, 2, 283. doi:10.7150/thno.3642

5. Mak, K. F.; Lee, C.; Hone, J.; Shan, J.; Heinz, T. F. Phys. Rev. Lett. 2010, 105, 136805. doi:10.1103/PhysRevLett.105.136805

6. Scheuschner, N.; Ochedowski, O.; Schleberger, M.; Maultzsch, J. Phys. Status Solidi B 2012, 1249, 2644. doi:10.1002/pssb.201200389

7. Plechinger, G.; Schrettenbrunner, F.-X.; Eroms, J.; Weiss, D.; Schüller, C.; Korn, T. Phys. Status Solidi RRL 2012, 6, 126. doi:10.1002/pssr.201105589

8. Tonndorf, P.; Schmidt, R.; Böttger, P.; Zhang, X.; Börner, J.; Liebig, A.; Albrecht, M.; Kloc, C.; Gordan, O.; Zahn, D. R. T.;

Michaelis de Vasconcellos, S.; Bratschlitsch, R. Opt. Express 2013, 21 , 4908. doi:10.1364/OE.21.004908

9. Cappelluti, E.; Roldan, R.; Silva-Guillén, J. A.; Ordejón, P.; Guinea, F. Phys. Rev. B 2013, 88, 075409. doi:10.1103/PhysRevB.88.075409
10. Castellanos-Gomez, A.; Poot, M.; Steele, G. A.; van der Zant, H. S. J.; Agrait, N.; Rubio-Bollinger, G. Adv. Mater. 2012, 24, 772. doi:10.1002/adma.201103965

11. Bertolazzi, S.; Brivio, J.; Kis, A. ACS Nano 2011, 5, 9703. doi:10.1021/nn203879f

12. Radisavljevic, B.; Radenovic, A.; Brivio, J.; Giacometti, V.; Kis, A. Nat. Nanotechnol. 2011, 6, 147. doi:10.1038/nnano.2010.279

13. Lembke, D.; Kis, A. ACS Nano 2012, 6, 10070. doi:10.1021/nn303772b

14. Yu, Y.; Li, C.; Liu, Y.; Su, L.; Zhang, Y.; Cao, L. Sci. Rep. 2013, 3 , No. 1866. doi:10.1038/srep01866

15. Zhan, Y.; Liu, Z.; Najmaei, S.; Ajayan, P. M.; Lou, J. Small 2012, 8, 966. doi:10.1002/smll.201102654

16. van der Zande, A. M.; Huang, P. Y.; Chenet, D. A.; Berkelbach, T. C.; You, Y.; Lee, G.-H.; Heinz, T. F.; Reichman, D. R.; Muller, D. A.; Hone, J. C. Nat. Mater. 2013, 12, 554. doi:10.1038/nmat3633

17. Wang, H.; Yu, L.; Lee, Y.-H.; Shi, Y.; Hsu, A.; Chin, M. L.; Li, L.-J.; Dubey, J.; Kong, M.; Palacios, T. Nano Lett. 2012, 12, 4674. doi:10.1021/nl302015v

18. Buscema, M.; Barkelid, M.; Zwiller, V.; van der Zant, H. S. J.; Steele, G. A.; Castellanos-Gomez, A. Nano Lett. 2013, 13, 358. doi:10.1021/nl303321g

19. Pu, J.; Yomogida, Y.; Liu, K.-K.; Li, L.-J.; Iwasa, Y.; Takenobu, T. ACS Nano 2012, 12, 4013. doi:10.1021/nl301335q

20. Radisavljevic, B.; Whitwick, M. B.; Kis, A. Appl. Phys. Lett. 2012, 101, 043103. doi:10.1063/1.4738986

21. Perkins, F. K.; Friedman, A. L.; Cobas, E.; Campbell, P. M.; Jernigan, G. G.; Jonker, B. T. Nano Lett. 2013, 13, 668. doi:10.1021/nl3043079

22. Late, F. J.; Liu, B.; Ramakrishna Matte, H. S. S.; David, V. P.; Rao, C. N. R. ACS Nano 2012, 6, 5635. doi:10.1021/nn301572c

23. Das, S.; Chen, H.-Y.; Penumatcha, A. V.; Appenzeller, J. Nano Lett. 2013, 13, 100. doi:10.1021/nl303583v 
24. Li, S.-L.; Wakabayashi, K.; Xu, Y.; Nakaharai, S.; Komatsu, K.; Li, W.-W.; Lin, A.; Aparecido-Ferreira, Y.-L.; Tsukagoshi, K. Nano Lett. 2013, 13, 3546. doi:10.1021/nl4010783

25. Fontana, M.; Deppe, T.; Boyd, A. K.; Rinzan, M.; Liu, A. Y.; Paranjape, M.; Barbara, P. Sci. Rep. 2013, 3, No. 1634. doi:10.1038/srep01634

26. Chen, W.; Santos, E. J. G.; Zhu, W.; Kaxiras, E.; Zhang, Z. Nano Lett. 2013, 13, 509. doi:10.1021/nl303909f

27. Bao, W.; Cai, X.; Kim, D.; Sridhara, K.; Fuhrer, M. S. Appl. Phys. Lett. 2013, 102, 042104. doi:10.1063/1.4789365

28. Hao, G.; Huang, Z.; Liu, Y.; Qi, X.; Ren, L.; Peng, X.; Yang, L.; Wei, X.; Zhong, J. AIP Adv. 2013, 3, 042125. doi:10.1063/1.4802921

29. Li, Y.; Xu, C.-Y.; Zhen, L. Appl. Phys. Lett. 2013, 102, 143110. doi:10.1063/1.4801844

30. Rad, M. A.; Ibrahim, K.; Mohamed, K. Superlattices Microstruct. 2012, 51, 597. doi:10.1016/j.spmi.2012.03.002

31. Gatzert, C.; Blakers, A. W.; Deenapanray, N. K.; Macdonald, D.; Auret, F. D. J. Vac. Sci. Technol., A 2006, 24, 1857. doi:10.1116/1.2333571

32. Novoselov, K. S.; Jiang, G.; Schedin, F.; Booth, T. J.; Khotkevich, V. V.; Morozov, S. V.; Geim, A. K. Proc. Natl. Acad. Sci. U. S. A. 2005, 102, 10451. doi:10.1073/pnas.0502848102

33. Castellanos-Gomez, A.; Agraït, N.; Rubio-Bollinger, G. Appl. Phys. Lett. 2010, 96, 213116. doi:10.1063/1.3442495

34. Lee, C.; Yan, H.; Brus, L. E.; Heinz, T. F.; Hone, J.; Ryu, S. ACS Nano 2010, 4, 2695. doi:10.1021/nn1003937

35. Nonnenmacher, M.; O’Boyle, M. P.; Wickramasinghe, H. K. Appl. Phys. Lett. 1991, 58, 2921. doi:10.1063/1.105227

36. Kitamura, S.; Iwatsuki, M. Appl. Phys. Lett. 1998, 72, 3154. doi:10.1063/1.121577

37. Glatzel, T.; Sadewasser, S.; Lux-Steiner, M. Ch. Appl. Surf. Sci. 2003, 210, 84. doi:10.1016/S0169-4332(02)01484-8

38. Sadewasser, S.; Glatzel, T.; Shikler, R.; Rosenwaks, Y.; Lux-Steiner, M. Ch. Appl. Surf. Sci. 2003, 210, 32. doi:10.1016/S0169-4332(02)01475-7

39. Rosenwaks, Y.; Shikler, R.; Glatzel, T.; Sadewasser, S. Phys. Rev. B 2004, 70, 085320. doi:10.1103/PhysRevB.70.085320

40. Zerweck, U.; Loppacher, C.; Otto, T.; Grafström, S.; Eng, L. M. Phys. Rev. B 2005, 71, 125424. doi:10.1103/PhysRevB.71.125424

41. Elias, G.; Glatzel, T.; Meyer, E.; Schwarzman, A.; Boag, A.; Rosenwaks, Y. Beilstein J. Nanotechnol. 2011, 2, 252. doi:10.3762/bjnano.2.29

42. Bertrand, P. A. Phys. Rev. B 1991, 44, 5745. doi:10.1103/PhysRevB.44.5745

43. Li, H.; Zhang, Q.; Yap, C. C. R.; Tay, B. K.; Edwin, T. H. T.; Olivier, A.; Baillargeat, D. Adv. Funct. Mater. 2012, 22, 1385. doi:10.1002/adfm.201102111

44. Conley, H. J.; Wang, B.; Ziegler, J. I.; Haglund, R. F., Jr..; Pantelides, S. T.; Pantelides, T.; Bolotin, K. I. Nano Lett. 2013, 13, 3626. doi:10.1021/nl4014748

45. Zhu, C. R.; Wang, G.; Lui, B. L.; Marie, X.; Quiao, X. F.; Zhang, X.; Wu, X. X.; Fan, H.; Tan, P. H.; Amand, T.; Urbaszek, B. Phys. Rev. B 2013, 88, 121301(R). doi:10.1103/PhysRevB.88.121301

46. Chakraborty, B.; Bera, A.; Muthu, D. V. S.; Bhowmick, S.; Waghmare, U. V.; Sood, A. K. Phys. Rev. B 2012, 85, 161403(R). doi:10.1103/PhysRevB.85.161403

47. Michaelson, H. B. J. Appl. Phys. 1977, 48, 4729. doi:10.1063/1.323539

48. Orf, N. D.; Baikie, I. D.; Shapira, O.; Fink, Y. Appl. Phys. Lett. 2009, 94, 113504. doi:10.1063/1.3089677
49. Ochedowski, O.; Kleine Bussmann, B.; Ban d'Etat, B.; Lebius, H.; Schleberger, M. Appl. Phys. Lett. 2013, 102, 153103. doi:10.1063/1.4801973

50. Giovannetti, G.; Khmyakov, P. A.; Brocks, G.; Karpan, V. M.; van den Brink, J.; Kelly, P. J. Phys. Rev. Lett. 2008, 101, 026803. doi:10.1103/PhysRevLett.101.026803

51. Domanski, A. L.; Sengupta, E.; Bley, K.; Untch, M. B.; Weber, S. A. L.; Landfester, K.; Weiss, C. K.; Butt, H.-J.; Berger, R. Langmuir 2012, 28, 13892. doi:10.1021/la302451h

52. Guo, L. Q.; Zhao, X. M.; Bai, Y.; Qiao, L. J. Appl. Surf. Sci. 2012, 258, 9087. doi:10.1016/j.apsusc.2012.06.003

53. Chen, J.-H.; Jang, C.; Adam, S.; Fuhrer, M. S.; Williams, E. D.; Ishigami, M. Nat. Phys. 2008, 4, 377. doi:10.1038/nphys935

54. Ryu, S.; Liu, L.; Berciaud, S.; Yu, Y.-J.; Liu, H.; Kim, P.; Flynn, G. W.; Brus, L. E. Nano Lett. 2010, 10, 4944. doi:10.1021/nl1029607

55. Molina-Sánchez, A.; Wirtz, L. Phys. Rev. B 2011, 84, 155413. doi:10.1103/PhysRevB.84.155413

56. Scheuschner, N.; Ochedowski, O.; Kaulitz, A.-M.; Gillen, R.; Schleberger, M.; Maultzsch, J. arXiv.org 2013, No. arXiv:1311.5824. [cond-mat.mtrl-sci].

57. Burson, K. M.; Cullen, W. G.; Adam, S.; Dean, C. R.; Watanabe, K.; Taniguchi, T.; Kim, P.; Fuhrer, M. S. Nano Lett. 2013, 13, 3576. doi:10.1021/nl4012529

58. Yamamato, M.; Einstein, T. L.; Fuhrer, M. S.; Cullen, W. G. ACS Nano 2012, 6, 8335. doi:10.1021/nn303082a

59. Castellanos-Gomez, A.; Roldán, R.; Cappelluti, E.; Buscema, M.; Guinea, F.; van der Zant, H. S. J.; Steele, G. A. Nano Lett. 2013, 13, 5361. doi:10.1021/nl402875m

60. McGovern, I. T.; Williams, R. H. Surf. Sci. 1974, 46, 427 doi:10.1016/0039-6028(74)90318-5

61. McMenamin, J. C.; Spicer, W. E. Phys. Rev. B 1977, 16, 5474. doi:10.1103/PhysRevB.16.5474

62. Kamaratos, M.; Papageorgopoulos, C. A. Surf. Sci. 1986, 178, 865. doi:10.1016/0039-6028(86)90362-6

63. Yun, J.-M.; Noh, Y.-J.; Yeo, J.-S.; Go, Y.-J.; Na, S.-I.; Jeong, H.-G.; Kim, J.; Lee, S.; Kim, S.-S.; Koo, H.-Y.; Kim, T.-W.; Kim, D.-Y. J. Mater. Chem. C 2013, 1, 3777. doi:10.1039/c3tc30504j

\section{License and Terms}

This is an Open Access article under the terms of the Creative Commons Attribution License (http://creativecommons.org/licenses/by/2.0), which permits unrestricted use, distribution, and reproduction in any medium, provided the original work is properly cited.

The license is subject to the Beilstein Journal of Nanotechnology terms and conditions: (http://www.beilstein-journals.org/bjnano)

The definitive version of this article is the electronic one which can be found at: $\underline{\text { doi: } 10.3762 / \text { bjnano.5.32 }}$ 